\title{
Effects of Rebate on the Evolution of Cooperation in Spatial Social Dilemmas
}

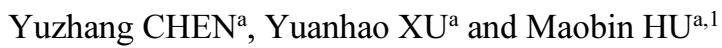

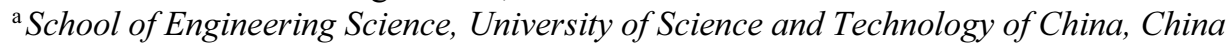

\begin{abstract}
Rebate, as a significant method to enhance cooperation between retailers and suppliers, has been widely applied among different commercial agencies. In this paper, we studied the effect of rebate on the evolution of cooperation in spatial social dilemmas. With a rebate mechanism, cooperators will share a part of payoff to cooperative neighbors. Simulation results shows that the rebate mechanism can promote cooperation significantly. When rebate mechanism works, the low-payoff boundary cooperators could receive extra rebates from the high-payoff cooperators in cooperator cluster. This helps cooperators defend the invasion of defectors. As a result, cooperators could survive and even dominate the system. The research can provide a new insight to explore the widespread cooperative behavior in the natural world.
\end{abstract}

Keywords. Cooperation, social dilemmas, rebate

\section{Introduction}

Cooperation widely exists in human society and biological system. However, individuals always try to maximize their profits in order to survive from the competitive world, which can't explain the ubiquitous cooperative behavior. Hence, the emergence of the cooperative behavior among selfishness individuals has attract great attention [1]. One of the commonly used models is the so-called evolutionary game [2,3]. The evolution means that the games are played recursively between individuals. After each generation, individuals can change their strategies (cooperation and defection) based their payoffs.

For well-mixed populations, cooperators can hardly survive in the population, and tends to be defectors with generations. In order to figure out the underlining mechanism of cooperators survive in real systems, the evolutionary games have been performed on systems with non-trivial topology structures, such as regular square lattice [4], scale-free networks [5], small-world networks [6], and so on [7]. This kind of evolutionary games are often called special evolutionary games, in which games are only played between the adjacent individuals.

In addition, many other mechanisms are also studied in the evolutionary game theory, such as reward [8], punishment [9], memory effect [10], strategy persistence [11], compassion effect [12], appropriate payoff aspiration [13], and so on. In general, the mechanisms that promote the emergence of cooperation can be divided into five

${ }^{1}$ Corresponding Author, Maobin HU, School of Engineering Science, University of Science and Technology of China, China; Email: humaobin@ustc.edu.cn 
categories: kin selection, group selection, network reciprocity, direct reciprocity and indirect reciprocity [14].

Here, we focus on a commonly used marketing strategy, namely rebate, in commercial world [15]. Rebate is a kind of payment by the means of reduction or refund on what has already been paid or contributed. Although this part of return seems to decrease the profit of a single transaction, the increment of order quantities could increase the retailers' profit in the long run. If the transaction is seen as cooperation between retailers and suppliers, the daily life experience can tell us that the rebate can enhance the cooperation between retailers and suppliers. However, how it affects the evolution of cooperation needs to be further discussed, which is the focus of this paper.

The remainder of this paper are organized as follows. The evolutionary game and the rebate mechanism are introduced in section 2 . In section 3 , we investigate how rebate affect the evolution of cooperation. Finally, we summarize our observations in section 4.

\section{Model Formulation}

The evolution of cooperation is performed on a $100 \times 100$ square lattice with periodic boundary conditions, and each site represents an individual. The prisoner's dilemma game (PDG) is applied to model the game process between the adjacent individuals.

In each generation, an individual's payoffs in the games with its four neighbors are dependent on their strategies (cooperation and defection). Cooperators will both gains reward $R$ under mutual cooperation, and defectors will both receive the punishment $P$ under mutual defection. When a cooperator encounters a defector, the cooperator will acquire the so-called sucker's payoff $S$ and the defector will acquire the temptation $T$. In particular, the payoffs of PDG satisfy the $T>R>P>S$. Without loss of generality, we set $R=1, P=0, S=0$ and $T=b$, where $b \in(1,2)$ characterizes the temptation of defectors.

At the beginning of the simulation, the individuals are designed as a cooperator or a defector with equal probability. Then, each individual play games with its four neighbors, respectively. The payoffs are calculated as above rules. The total payoff of an individual is defined as:

$$
P_{i}=\sum_{j \in \varphi} p_{i j}
$$

where $p_{i j}$ is the payoff of individual $i$ in the game with individual $j$, and the $\operatorname{sum} \varphi$ is over the neighbors of individual $i$.

As cooperators just receive the payoff from cooperator neighbors $(R=1)$ instead of defector neighbors $(S=0)$. For the rebate mechanism, we assume that cooperators participate in the rebate activity and only share their payoff to cooperator neighbors. A payoff aspiration level $\alpha$ is considered for cooperators to determine whether share their payoff to cooperator neighbors. If a cooperator's payoff satisfies $P_{i}>\alpha$, the rebate mechanism works and the payoff between cooperators will be redistributed as:

$$
\begin{aligned}
& P_{i}=\alpha \\
& P_{j}=P_{j}+\left(P_{i}-\alpha\right) / n
\end{aligned}
$$

i.e., cooperator $i$ share extra payoff $\left(P_{i}-\alpha\right)$ to their cooperator neighbors $j$ and the rest payoff (aspiration level $\alpha$ ) will be retained. Where $n$ denotes the amount of cooperator neighbors. Here $0 \leq \alpha \leq 4$ is the bound of the cooperator's payoff. Obviously, when $\alpha=4$, the model reduces to the classical PDG. 
After the rebate process, the cooperators' payoffs are redistributed. Then, individuals update their strategies based on their payoffs. Specially, an individual $i$ adopts a randomly chosen neighbor's (labelled $j$ ) strategy with probability.

$$
W_{i, j}=1 /\left(1+e^{\left(P_{i}-P_{\mathrm{j}}\right) / \tau}\right)
$$

where $\tau$ denotes the noise, and here $\tau=0.1$. Note that all individuals update their strategies synchronously.

\section{Simulation Results}

First, we observe the frequency of cooperation as a function of defectors' temptation $b$ under different aspiration level, which shown in figure 1 . When $\alpha=4$, the cooperators' payoff less than or equal to the aspiration level, the model reduces to the classical PDG. One can see that the cooperators can hardly survive in the population and vanishes just at $b=1.03$. Analogously, the frequency of cooperation remains almost unchanged when we set the aspiration level $\alpha=0$. It is because the payoff that cooperators shared $(n R-\alpha)$ and received $(n R)$ are equal when we set $\alpha=0$. As cooperators share extra payoff to their neighbors who adopt cooperate strategy, the cooperative behavior on square lattice could be promoted significantly $(\alpha=1,2,3)$. With the increment of $\alpha$, the frequency of cooperation increases at first and then decrease until the rebate mechanism loss efficacy. It is clear that an appropriate aspiration level with rebate mechanism could promote cooperation behavior under a certain game variable $b$. Therefore, it is necessary to discussed how game variable and aspiration level effect on the cooperation frequency in detail.

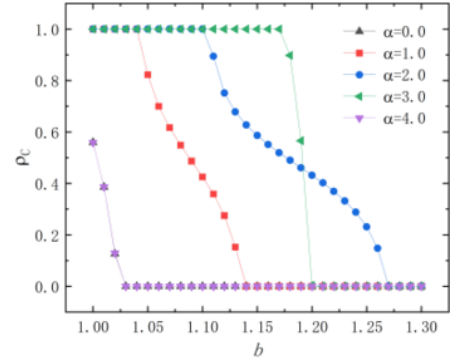

Figure 1. (Color Online) The cooperation frequency as a function of defect temptation $b$ (PDG) under different aspiration level.

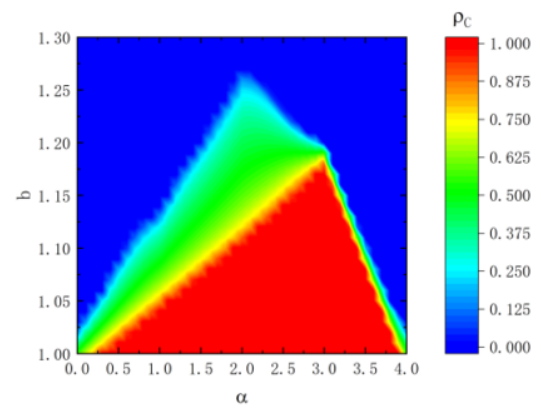

Figure 2. (Color Online) Contour map of the cooperation frequency $\rho_{c}$ on $b$ - $\alpha$ panel. 
To better explore the rebate mechanism on global perspective, we observe the frequency of cooperation $\rho_{c}$ on $b$ - $\alpha$ panel in figure 2. As we fixed aspiration level $\alpha$, with the increase of game variable $b$, the cooperation frequency remain decrease until all cooperators vanish in the system. On the other hand, as we fixed game variable $b$, with the increase of aspiration level $\alpha$, the cooperation frequency increase at first and then decrease. It is clearly that maximum value of cooperation frequency appears at the point $\alpha=3$. However, when $b>1.2$, cooperators can survive in the system at the point $\alpha=2$ instead of $\alpha=3$. There are two peak values of $\alpha$ appears in the contour map, one $(\alpha=2)$ denotes the maximum value of $b$ that cooperators can survive, another one $(\alpha=3)$ denotes the maximum value of $b$ that all individuals adopt cooperation strategy.

In order to figure out the evolutionary process, we observe the evolution of cooperation frequency in figure 3(a). The cooperation frequency decreases quickly at early stage and then defectors dominate the whole population when $\alpha=0,4$. Compare with traditional PDG $(\alpha=4)$, cooperators could survive in the system as we changed rebate parameter $(\alpha=1,2,3)$. Different from the traditional case, the cooperators could survive and even dominate the system. Although the final cooperation frequency reaches the maximum value when $\alpha=3$, more cooperators appears at early stage when $\alpha=1,2$.
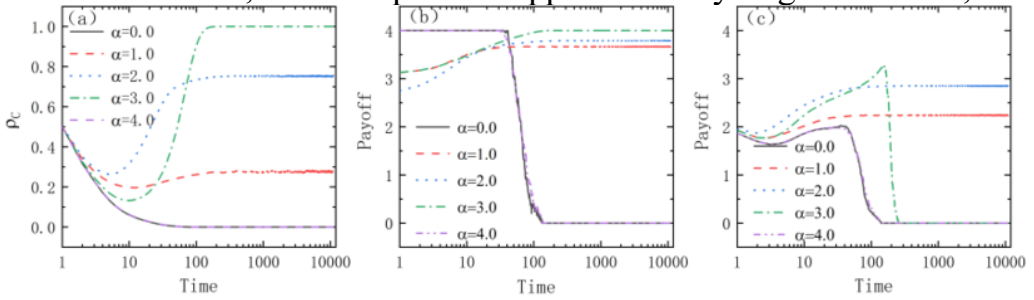

Figure 3. (Color Online) Dynamics of cooperation frequency and payoff: (a)the cooperation frequency, (b)the average payoff of pure cooperator clusters, (c) the average payoff of boundary cooperators. All results are observed at $b=1.12$.
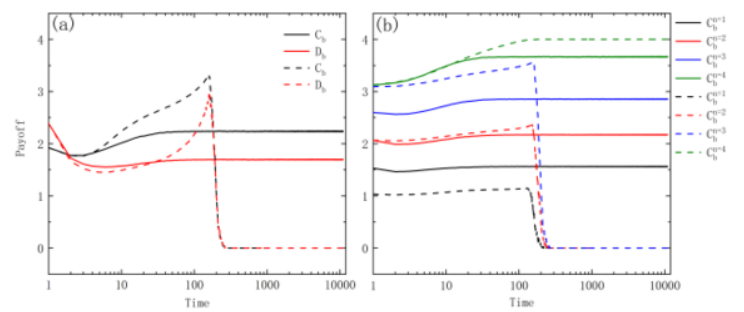

Figure 4. (Color Online) Dynamics of payoff: (a)the average payoff of boundary cooperator and boundary defectors, (b)the average payoff of cooperator with different amount of cooperator neighbors. Where $n$ denotes the amount of cooperator neighbors and $0 \leq n \leq 4$. For full line we set $\alpha=1$ and dotted line $\alpha=3$. All results are observed at $b=1.12$.

Trying to further understand the effect of rebate mechanism, we investigate the time series of individuals' payoff. We divide individuals into four categories: pure cluster cooperators $(C c)$, pure cluster defectors $(D c)$, boundary cooperators $(C b)$, boundary defectors $(D b)$. Especially, boundary cooperators have at least one defector neighbor, and boundary defectors have at least one cooperator neighbor. Figure 3(b) and (c) features the average payoff dynamics of $C c$ and $C b$ respectively. For $\alpha=0$ and $\alpha=4$, with the evolution of time, the payoff of $C c$ remains unchanged at early stage while the payoff of $C b$ changes a few. Then, due to all cooperators transfer into 
defectors, the payoff of $C c$ and $C b$ drop to 0 . For $\alpha=1,2,3$, we can observe the boundary cooperators gains more payoff than traditional case $(\alpha=4)$. Correspondingly, the decline of $P_{C c}$ can be observed in figure3(b). It means boundary cooperators could receive a rebate from cluster cooperators generally. As a result, boundary cooperators could defend the invasion of defectors and survive in the populations. In particular, at earlier stage, the payoff of boundary cooperators satisfies $P_{C c}^{\alpha=2}>P_{C c}^{\alpha=3}$. Therefore, more cooperators emerge in the system at earlier stage when $\alpha=2$ rather than $\alpha=3$ can be seen in figure 3(a).

However, for $\alpha=1$ and $\alpha=3$, the average payoff of boundary cooperators is very close which can't explain why more cooperators emerge at early stage when $\alpha=1$. Hence, we study the redistribution of individuals' payoff in detail. Figure 4 exhibits the evolution of different individuals' payoff at $b=1.12$. Initially, cooperators and defectors are random distributed on the square lattice, and most individuals are boundary players. Defectors acquire payoff from cooperator neighbors and the payoff satisfy $P_{D b}>P_{C b}$ (see Time $=0$ in figure 4(a)). Then, cooperators receive extra rebate to defend the invasion of defectors and the payoff turn into $P_{D b}>P_{C b}$ (see Time=10 in figure 4 (a)). We observed the cooperators' average payoff with different amount of cooperator neighbors in figure 4 (b). With the increment of cooperator neighbor $n$, the cooperators' payoff will increase as well. For cooperator have 2 or 4 cooperator neighbors, the average payoff $P_{C c}^{\alpha=1}$ and $P_{C c}^{\alpha=3}$ are approximately equal at early stage. The main difference between aspiration level $\alpha=1$ and $\alpha=3$ is the case of cooperator neighbor $n=1$ and $n=3$. For $\alpha=1$, we can see that $P_{C c}^{n=1}>1$ and $P_{C c}^{n=3}<3$. However, the payoff of cooperators satisfies $P_{C c}^{n=1} \approx 1$ and $P_{C c}^{n=3}>3$ when $\alpha=3$. Therefore, cooperator with one cooperator neighbor receive payoff from cooperator with three cooperator neighbors when $\alpha=1$. Cooperator only have one cooperator neighbor $(n=1)$ are highly risk to adopt defect strategy during strategy updating process. Compare $\alpha=1$ and $\alpha=3$, it is obvious that $P_{C c}^{n=1, \alpha=1}>P_{C c}^{n=1, \alpha=3}$ which lead to the higher cooperation frequency of $\alpha=1$ at early stage.

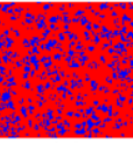

(a1)

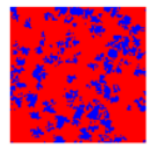

(а2)

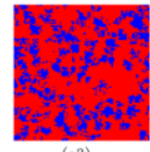

(a3)

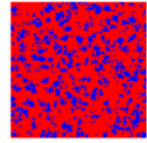

(b1)

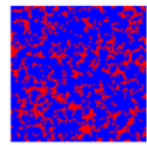

(b2)

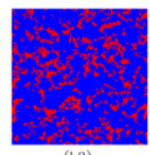

(b3)

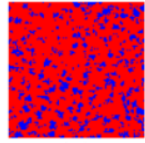

(c1)

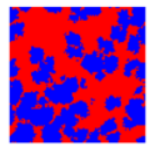

(c2)

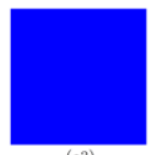

(c3)

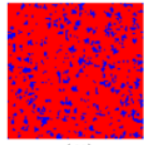

(d1)

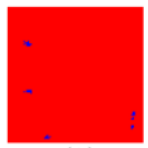

(d2)

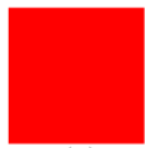

(d3)

Figure 5. (Color Online) Spatial distribution of individuals under different values of $\alpha$. Here blue lattice denotes the cooperators and red lattice denotes the defectors. (a) $\alpha=1$, (b) $\alpha=2$, (c) $\alpha=3$, (d) $\alpha=4$. Rows from top to bottom: (1) Time=5, (2) Time $=50,(3)$ Time $=500$. All results are obtained at $b=1.12$ on square lattice.

Finally, it is necessary to investigate the spatial distributions of individuals on square lattice. As shown in figure 5(d), defectors dominate the system for the traditional case $(\alpha=4)$. Nevertheless, the cooperative behaviour could be promoted significantly as we considering rebate mechanism. Cooperators form clusters to defend the invasion of 
defectors can be observed in figure 5 (a), (b), (c). Generally speaking, with the evolution time, the size and quantity of cooperator clusters remain increase. When $\alpha=3$, larger cooperator clusters appear in the population (figure 5(c2)) and all individuals adopt cooperate strategy eventually. For Time $=5$ and Time $=50$, more cooperators emerge in the square lattice when $\alpha=2$ rather than $\alpha=1,3$, which consistent with what we discussed above.

\section{Conclusion}

In summary, we have studied how the rebate mechanism affects the evolution of cooperation in the spatial social dilemmas. By the rebate mechanism, cooperators share their payoff to the neighbors who adopt cooperative strategy. The rebate payoff between cooperators can be adjusted by the aspiration level.

Simulation results implies that the rebate mechanism promote the cooperative behaviour significantly. As the rebate mechanism works, low-payoff boundary cooperators could receive the rebate from high-payoff pure cooperator clusters. And cooperators form clusters to defend the invasion of defectors. With the increase of aspiration level, the cooperation frequency increase at first and then decrease to traditional PDG. For a certain game variable, there is an optimal value of aspiration level to promote cooperative behaviour. Our work could provide a new perspective to understanding the emergence and maintenance of cooperation.

\section{Acknowledgments}

This work is supported by the National Natural Science Foundation of China (No.12072340).

\section{References}

[1] Nowak, M. A. and May, R. M., Evolutionary games and spatial chaos. Nature, 1992, 359.6398: 826-829.

[2] Lieberman, E., Hauert, C., and Nowak, M. A., Evolutionary dynamics on graphs. Nature, 2005, 433.7023: 312-316.

[3] Wu, Z. X., Xu, X. J., Huang, Z. G., Wang, S. J., and Wang, Y. H., Evolutionary prisoner's dilemma game with dynamic preferential selection. Physical Review E, 2006, 74.2: 021107.

[4] Perc, M., Chaos promotes cooperation in the spatial prisoner's dilemma game. EPL (Europhysics Letters), 2006, 75.6: 841.

[5] Santos, F. C., and Pacheco, J. M., Scale-free networks provide a unifying framework for the emergence of cooperation. Physical review letters, 2005, 95.9: 098104.

[6] Chen X. and Wang L., Promotion of cooperation induced by appropriate payoff aspirations in a smallworld networked game. Physical Review E, 2008, 77.1: 017103.

[7] Vukov, J., Szabó, G., and Szolnoki, A., Cooperation in the noisy case: Prisoner's dilemma game on two types of regular random graphs. Physical Review E, 2006, 73.6: 067103.

[8] Wang, Z., Szolnoki, A., and Perc, M., Rewarding evolutionary fitness with links between populations promotes cooperation. Journal of theoretical biology, 2014, 349: 50-56.

[9] Yang, H. X., and Rong, Z., Mutual punishment promotes cooperation in the spatial public goods game. Chaos, Solitons \& Fractals, 2015, 77: 230-234.

[10] Qin, S. M., Chen, Y., Zhao, X. Y., and Shi, J., Effect of memory on the prisoner's dilemma game in a square lattice. Physical Review E, 2008, 78.4: 041129. 
[11] Huang, C. W., and Dai, Q. L., Persistence paves the way for cooperation in evolutionary games. EPL (Europhysics Letters), 2017, 118.2: 28002.

[12] Li, Y., Zhang, J., and Perc, M., Effects of compassion on the evolution of cooperation in spatial social dilemmas. Applied Mathematics and Computation, 2018, 320: 437-443.

[13] Liu, R. R., Jia, C. X., and Rong, Z., Effects of enhancement level on evolutionary public goods game with payoff aspirations. Applied Mathematics and Computation, 2019, 350: 242-248.

[14] Nowak, M. A., Five rules for the evolution of cooperation. science, 2006, 314.5805: 1560-1563.

[15] Brown, A., Chou, M. C., and Tang, C. S., Christopher S. The implications of pooled returns policies. International Journal of Production Economics, 2008, 111.1: 129-146. 